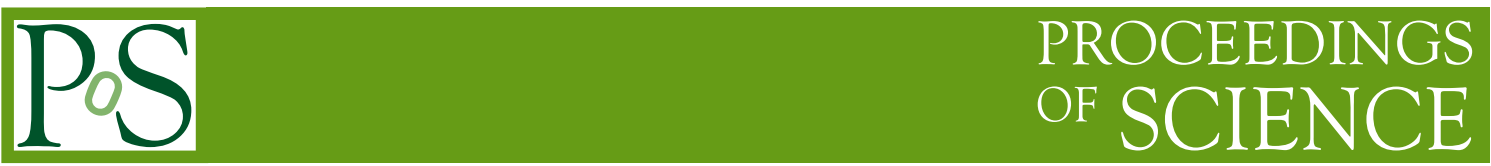

Holographic superconductors at low temperatures

\title{
George Siopsis*i
}

Department of Physics and Astronomy

The University of Tennessee

Knoxville, TN 37996-1200

U.S.A.

E-mail: Siopsisetennessee.edu

I develop analytic tools in order to study the properties of holographic superconductors in an arbitrary dimension $d$ with dual blck holes with scalar hair of mass $m$ and charge $q$. I discuss the low temperature limit where numerical methods are plagued by instabilities. In the large $q$ (probe) limit, I show that the energy gap diverges as a (negative) power of the temperature for scaling dimensions below the Breitenlohner-Freedman (BF) bound $(\Delta<d / 2)$. At the BF bound $(\Delta=d / 2)$, the condensate also diverges at low temperatures as $|\ln T|^{\delta}$, where $\delta$ depends on the dimension. This mild divergence was missed in earlier numerical studies. I calculate the conductivity analytically and show that at low temperatures, it has spikes whose locations are determined by the zeroes of the Airy function in $2+1$ dimensions and of the digamma function in 3+1 dimensions. The analytic results are in good agreement with numerical results whenever the latter are available. I also discuss the small $q$ (extremal) limit concentrating on the quantum critical point $q_{c}$ at which the critical temperature $T_{c}$ vanishes. I show that as $q \rightarrow q_{c}\left(T_{c} \rightarrow 0\right)$, the energy gap diverges.

Proceedings of the Corfu Summer Institute 2011 School and Workshops on Elementary Particle Physics and Gravity

September 4-18, 2011

Corfu, Greece

\footnotetext{
* Speaker.

${ }^{\dagger}$ Research supported in part by the US Department of Energy under grant DE-FG05-91ER40627.
} 


\section{Introduction}

The extension of the AdS/CFT correspondence [1] to an abelian gauge group has been studied extensively in recent years. Once it the abelian symmetry is broken outside of a black hole in AdS space, scalar hair can form whose dual is a (holographic) superconductor on the boundary of AdS space [月, 3, 4, 5]. These systems exhibit several characteristics seen in the real world, thus offering a new theoretical tool in the exploration of strongly coupled superconductors.

The study of holographic superconductors involves the solution of non-linear field equations. These are very hard to solve in general and numerical methods often fail, especially as one approaches zero temperature. The latter is important to be able to probe in order to understand the ground state of these systems. It is important to develop analytic tools that tackle the solutions of the field equations and this is what I discuss here. I am especially interested in understanding the low temperature regime in two different limits: small chemical potential (probe limit, whose dual is a Schwarzschild black hole) [6, 7], and large chemical potential (dual to near-extremal Reissner-Nordström black holes) [8].

In the probe limit, I solve the field equations analytically at low temperatures for different values of the scaling dimension $\Delta$. For $\Delta<\frac{d}{2}$ (below the scaling dimension of the BreitenlohnerFreedman (BF) bound), the energy gap diverges as a (negative) power of the temperature, signaling the breakdown of the probe approximation in the zero temperature limit. I discuss how this is corrected by including next-to-leading order terms. At the BF bound $\left(\Delta=\frac{d}{2}\right.$, the energy gap diverges as $|\ln T|^{\delta}$, where $\delta$ is a constant that I compute and depends on the dimension of spacetime [7]. This is a very mild divergence which explains why it was not detected in earlier numerical studies [4].

When back reaction to the metric is included, the effective potential associated with the electromagnetic perturbation that determines the conductivity has a finite height. This results in a finite number of quasinormal modes [ 9 , 9]. As one approaches the probe limit, the height of the potential increases with an attendant increase in the number of modes. I show that the latter approach the real axis as the temperature is lowered at frequencies that I compute analytically. In $2+1(3+1)$ dimensions they are given in terms of the zeroes of the Airy (digamma) function [7].

It should be pointed out that, even though I probe the low temperature regime, I do not have access to the zero temperature state in the probe limit (for scaling dimensions $\Delta \leq \frac{d}{2}$ ). This is because for a given charge of the scalar field, there is a lower bound on the temperature below which the probe approximation breaks down. Thus, even though it is often possible to take a "zero temperature" limit of analytic expressions, the limit itself is unphysical. It is, however, useful for computational purposes, as physical low-temperature systems (obtained when backreaction to the bulk metric is taken into account) are close to it.

In the case of large chemical potential (near-extremal limit of the dual black hole), I show analytically that there is a quantum critical point at which the critical temperature vanishes. As one approaches this critical point, the energy gap diverges [8].

The discussion is organized as follows. In section 2 , I review the field equations and calculate the critical temperature in the probe limit. In section 3, I discuss the low temperature regime in the probe limit. In section 4 , I calculate the conductivity analytically at low temperatures in the probe 
limit. In section 5, I discuss the analytic solution of the field equations near extremality. Finally, section 6 contains my concluding remarks.

\section{Hairy black holes}

I am interested in the dynamics of a scalar field of mass $m$ and electric charge $q$ coupled to a $U(1)$ vector potential in the backgound of a $d+1-$ dimensional AdS black hole. The action is

$$
S=\int d^{d+1} \sqrt{-g}\left[\frac{R-2 \Lambda}{16 \pi G}-\frac{1}{4} F_{\mu \nu} F^{\mu v}-\left|\left(\partial_{\mu}-i q A_{\mu}\right) \Psi\right|^{2}-m^{2}|\Psi|^{2}\right]
$$

where $F=d A$. I shall adopt units in which $\Lambda=-\frac{d(d-1)}{2}, 16 \pi G=1$.

To find a solution of the field equations, consider the metric ansatz

$$
d s^{2}=\frac{1}{z^{2}}\left[-f(z) e^{-\chi(z)} d t^{2}+d \vec{x}^{2}+\frac{d z^{2}}{f(z)}\right]
$$

where $\vec{x} \in \mathbb{R}^{d-1}$, representing an AdS black hole of planar horizon. The AdS boundary is at $z=0$. I shall choose units so that the horizon is at $z=1$, and therefore require $f(1)=0$. This is possible because of the scaling symmetries of the system and can be done without loss of generality as long as one is careful to only consider physical quantities which are scale invariant.

The Hawking temperature is

$$
T=-\frac{f^{\prime}(1)}{4 \pi} e^{-\chi(1) / 2}
$$

Also assume that the scalar field is a real function $\Psi(z)$, and the electromagnetic potential is the electrostatic potential, $A=\Phi(z) d t$. The resulting field equations are [3]

$$
\begin{aligned}
\Psi^{\prime \prime}+\left[\frac{f^{\prime}}{f}-\frac{\chi^{\prime}}{2}-\frac{d-1}{z}\right] \Psi^{\prime}+\left[\frac{q^{2} \Phi^{2} e^{\chi}}{f^{2}}-\frac{m^{2}}{z^{2} f}\right] \Psi & =0 \\
\Phi^{\prime \prime}+\left[\frac{\chi^{\prime}}{2}-\frac{d-3}{z}\right] \Phi^{\prime}-\frac{2 q^{2} \Psi^{2}}{z^{2} f} \Phi & =0 \\
-\frac{d-1}{2} \chi^{\prime}+z \Psi^{\prime 2}+\frac{z q^{2} \Phi^{2} \Psi^{2}}{f^{2}} e^{\chi} & =0 \\
\frac{f}{2} \Psi^{\prime 2}+\frac{z^{2}}{4} \Phi^{\prime 2} e^{\chi}-\frac{d-1}{2} \frac{f^{\prime}}{z}+\frac{d(d-1)}{2} \frac{f-1}{z^{2}}+\frac{m^{2} \Psi^{2}}{2 z^{2}}+\frac{q^{2} \Psi^{2} \Phi^{2} e^{\chi}}{2 f} & =0
\end{aligned}
$$

where $z \in[0,1]$ ( $z=1$ is the horizon, whereas $z=0$ is the boundary).

I start by discussing the solution of this system of non-linear equations in the limit of large $q$ (probe limit). The other interesting limit (small $q$, corresponding to large chemical potential) will be discussed later. 
To find the solution for large $q$, expand the fields as series in $1 / q$,

$$
\begin{aligned}
& \Psi=\frac{1}{q}\left[\Psi_{0}+\Psi_{1} \frac{1}{q^{2}}+\ldots\right] \\
& \Phi=\frac{1}{q}\left[\Phi_{0}+\Phi_{1} \frac{1}{q^{2}}+\ldots\right] \\
& f=f_{0}+f_{1} \frac{1}{q^{2}}+\ldots \\
& \chi=\chi_{0}+\chi_{1} \frac{1}{q^{2}}+\ldots
\end{aligned}
$$

First, consider the zeroth order system $(q \rightarrow \infty)$, and then add first-order $\left(\mathscr{O}\left(1 / q^{2}\right)\right)$ corrections in order to obtain a physically sensible system.

Near the boundary $(z \rightarrow 0)$, we have $f \rightarrow 1, \chi \rightarrow 0$ and so

$$
\Psi \approx \Psi^{( \pm)} z^{\Delta_{ \pm}}, \quad \Phi \approx \mu-\rho z^{d-2}, \quad \Delta_{ \pm}=\frac{d}{2} \pm \sqrt{\frac{d^{2}}{4}+m^{2}}
$$

While a linear combination of asymptotics is allowed by the field equations, it turns out that any such combination is unstable [10]. However, if the horizon has negative curvature, such linear combinations lead to stable configurations in certain cases [11].

Thus, the system is labeled uniquely by the dimension $\Delta=\Delta_{ \pm}$. The mass of the scalar field is bounded from below by the BF bound,

$$
m^{2} \geq-\frac{d^{2}}{4}
$$

There appears to be a quantum phase transition at $m^{2}=0$. There is also a unitarity bound that requires $\Delta>\frac{d-2}{2} . \mu(\rho)$ is the chemical potential (charge density) of the dual theory on the boundary. The leading coefficient in the expansion of the scalar yields vacuum expectation values of operators of dimension $\Delta_{ \pm}$,

$$
\left\langle\mathscr{O}_{\Delta_{ \pm}}\right\rangle=\sqrt{2} \Psi^{( \pm)}
$$

The field equations admit non-vanishing solutions for the scalar below a critical temperature $T_{c}$ where these operators condense.

Define for convenience

$$
\Psi(z)=\frac{1}{\sqrt{2} q} b^{\Delta} z^{\Delta} F(z), \quad b=\left\langle q \mathscr{O}_{\Delta}\right\rangle^{1 / \Delta}
$$

with $F(0)=1$.

Above the critical temperature $\left(T \geq T_{c}\right)$, we have $\Psi=0$, therefore the field equations are solved by the AdS Reissner-Nordström (RN) black hole with flat horizon,

$$
f(z)=1-\left(1+\frac{(d-2) \rho^{2}}{4}\right) z^{d}+\frac{(d-2) \rho^{2}}{4} z^{2(d-1)}, \quad \chi(z)=0, \Phi(z)=\rho\left(1-z^{d-2}\right),
$$

and Hawking temperature (divided by an appropriate factor for a scale-invariant quantity)

$$
\frac{T}{(q \rho)^{1 /(d-1)}}=\frac{d}{4 \pi(q \rho)^{1 /(d-1)}}\left[1-\frac{(d-2)^{2} \rho^{2}}{4 d}\right] .
$$


At the critical temperature, the scalar field equation is in the RN background with $\rho=\rho_{c}$

$$
F^{\prime \prime}+\left[\frac{f^{\prime}}{f}+\frac{2 \Delta+1-d}{z}\right] F^{\prime}+\left[\Delta \frac{(d-\Delta)(1-f)+z f^{\prime}}{z^{2} f}+q^{2} \rho_{c}^{2} \frac{\left(1-z^{d-2}\right)^{2}}{f^{2}}\right] F=0
$$

For a given $q, \rho_{c}$ is an eigenvalue.

To solve for large $q$ (probe limit), expand

$$
F=F_{0}+F_{1} \frac{1}{q^{2}}+\ldots, \quad \rho=\frac{1}{q}\left[\rho_{0}+\rho_{1} \frac{1}{q^{2}}+\ldots\right]
$$

At zeroth order ( $q \rightarrow \infty$ limit), the RN background turns into an AdS Schwarzschild black hole of flat horizon, so

$$
f_{0}(z)=1-z^{d}, \quad \chi_{0}=0 .
$$

Therefore, the scalar field equation at the critical temperature in the probe limit reads

$$
-F_{0}^{\prime \prime}+\frac{1}{z}\left[\frac{d}{1-z^{d}}-2 \Delta-1\right] F_{0}^{\prime}+\Delta^{2} \frac{z^{d-2}}{1-z^{d}} F_{0}=\rho_{0 c}^{2} \frac{\left(1-z^{d-2}\right)^{2}}{\left(1-z^{d}\right)^{2}} F_{0} .
$$

The eigenvalue $\rho_{0 c}^{2}$ minimizes the expression

$$
\rho_{0 c}^{2}=\frac{\int_{0}^{1} d z z^{2 \Delta-d+1}\left\{\left(1-z^{d}\right)\left[F_{0}^{\prime}(z)\right]^{2}+\Delta^{2} z^{d-2}\left[F_{0}(z)\right]^{2}\right\}}{\int_{0}^{1} d z z^{2 \Delta-d+1} \frac{\left(1-z^{d-2}\right)^{2}}{1-z^{d}}\left[F_{0}(z)\right]^{2}} .
$$

It can be estimated by substituting the trial function

$$
F_{0}=F_{\alpha}(z) \equiv 1-\alpha z^{d-1}
$$

which obeys the boundary conditions $F_{\alpha}(0)=1, F_{\alpha}^{\prime}(0)=0$ and $F_{\alpha}(1)$ is finite.

For $d=3$ and $\Delta=1,2$, one obtains, respectively,

$$
\rho_{0 c}^{2} \approx 1.27,17.3, \quad T_{c} \approx 0.225 \sqrt{q \rho}, 0.117 \sqrt{q \rho}
$$

in very good agreement with the exact $T_{c}=0.226 \sqrt{q \rho}, 0.118 \sqrt{q \rho}$.

For $\Delta=\frac{d}{2}$ (BF bound) and $d=3,4$, one obtains, respectively,

$$
\rho_{0 c}^{2} \approx 6.3,4.2, \quad T_{c} \approx 0.15 \sqrt{q \rho}, 0.2(q \rho)^{1 / 3}
$$

in very good agreement with the exact $T_{c}=0.15 \sqrt{q \rho}, 0.25(q \rho)^{1 / 3}$. In fig. 1, I extend the comparison to a range of the scaling dimension $\Delta$ for $d=3,4$ demonstrating the accuracy of the estimate (2.16) with the trial function (2.17) for the critical temperature.

Right below the critical temperature, a condensate forms. For an explicit analytic calculation, I shall concentrate on $d=3$, as the other cases are similar.

The Maxwell equation (Gauss's Law) in the probe limit reads

$$
\Phi_{0}^{\prime \prime}=\frac{\left\langle\mathscr{O}_{\Delta}\right\rangle^{2}}{2} \frac{z^{2(\Delta-1)} F_{0}^{2}(z)}{1-z^{3}} \Phi_{0}
$$



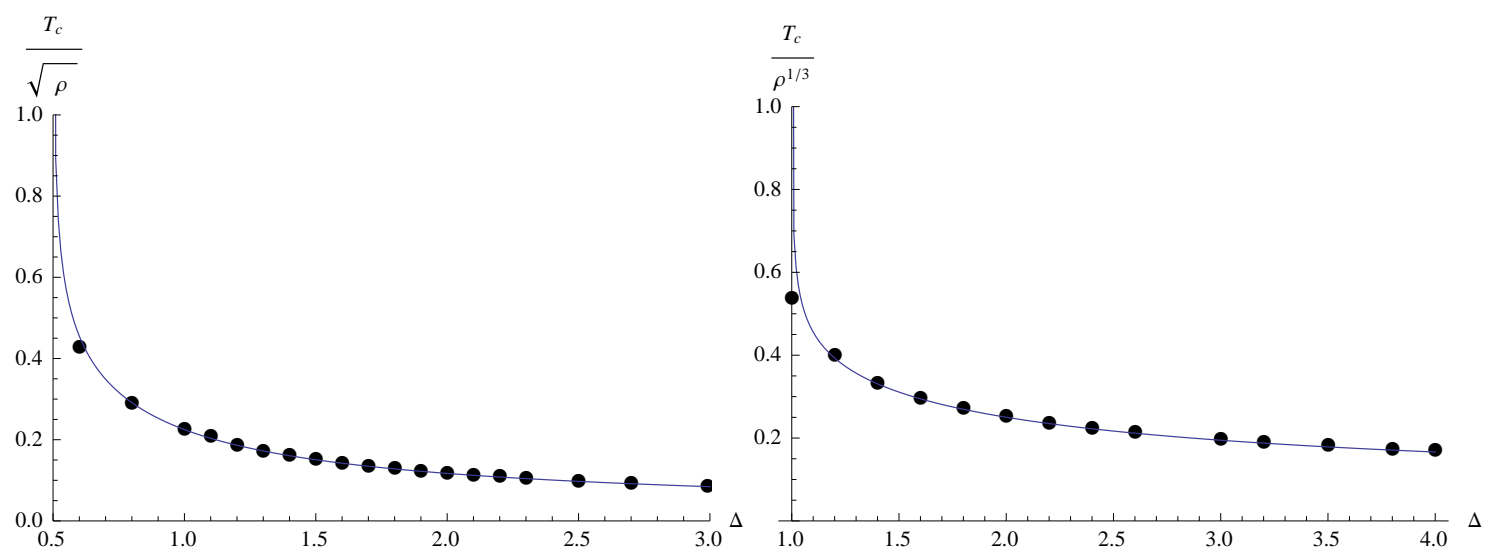

Figure 1: The critical temperature $T_{c} v s$ the scaling dimension $\Delta$ for $d=3$ (left panel) and $d=4$ (right panel). Data points represent exact values; solid line is obtained with trial function [6].

Right below $T_{c}$, the condensate $\left\langle\mathscr{O}_{\Delta}\right\rangle$ is small. If we write

$$
\Phi_{0}=\rho_{0 c}(1-z)+\frac{\left\langle\mathscr{O}_{\Delta}\right\rangle^{2}}{2} \delta \Phi_{0}+\ldots
$$

we have

$$
\delta \Phi_{0}^{\prime \prime} \approx \rho_{0 c} \frac{z^{2(\Delta-1)} F_{0}^{2}(z)}{1+z+z^{2}}
$$

Integrating, we obtain

$$
\rho=-\Phi_{0}^{\prime}(0) \approx \rho_{0 c}\left[1+\frac{\left\langle\mathscr{O}_{\Delta}\right\rangle^{2}}{2} \mathscr{C}\right], \quad \mathscr{C}=\int_{0}^{1} d z \frac{z^{2(\Delta-1)} F_{0}^{2}(z)}{1+z+z^{2}}
$$

therefore the condensate is

$$
\left\langle\mathscr{O}_{\Delta}\right\rangle \approx \gamma T_{c}^{\Delta} \sqrt{1-\frac{T}{T_{c}}}, \quad \gamma=\frac{2}{\sqrt{\mathscr{C}}}\left(\frac{4 \pi}{3}\right)^{\Delta} .
$$

Using trial functions, we obtain for $\Delta=1(2), \gamma \approx 11.4(133)$ to be compared with the exact $\gamma=$ $9.3(144)$.

Also notice from fig. 2 that $\gamma \rightarrow \infty$ as $\Delta \rightarrow 3$, indicating that $m^{2}=0$ is a quantum critical point.

\section{The probe limit at low temperature}

Next, I am interested in understanding analytically the behavior of the system at low temperatures. In the zero temperature limit $(T \rightarrow 0)$, we deduce from $(2.11)$ that $\rho \rightarrow \infty$. The condensate also diverges $(b \rightarrow \infty$, where $b$ is defined in (2.9)), since is it measured in units of the radius of the horizon (alternatively, observe that $b /(q \rho)^{1 /(d-1)}$ is a scale-invariant quantity, so both $\left.b, \rho \rightarrow \infty\right)$. Of interest is the behavior of scale-invariant quantities, such as the energy gap,

$$
\frac{E_{g}}{T_{c}}=\frac{\langle\mathscr{O}\rangle^{1 / \Delta}}{T_{c}} \sim \frac{b}{(q \rho)^{1 /(d-1)}}
$$




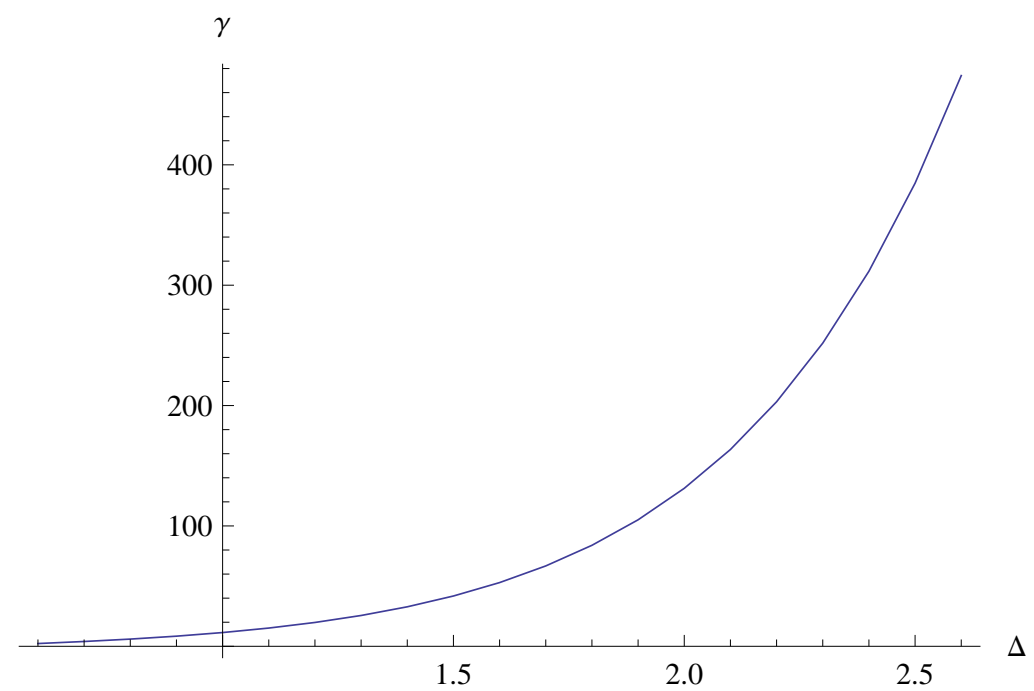

Figure 2: The parameter $\gamma$ of the condensate near $T_{c} v s . \Delta$ in $d=3$ [6].

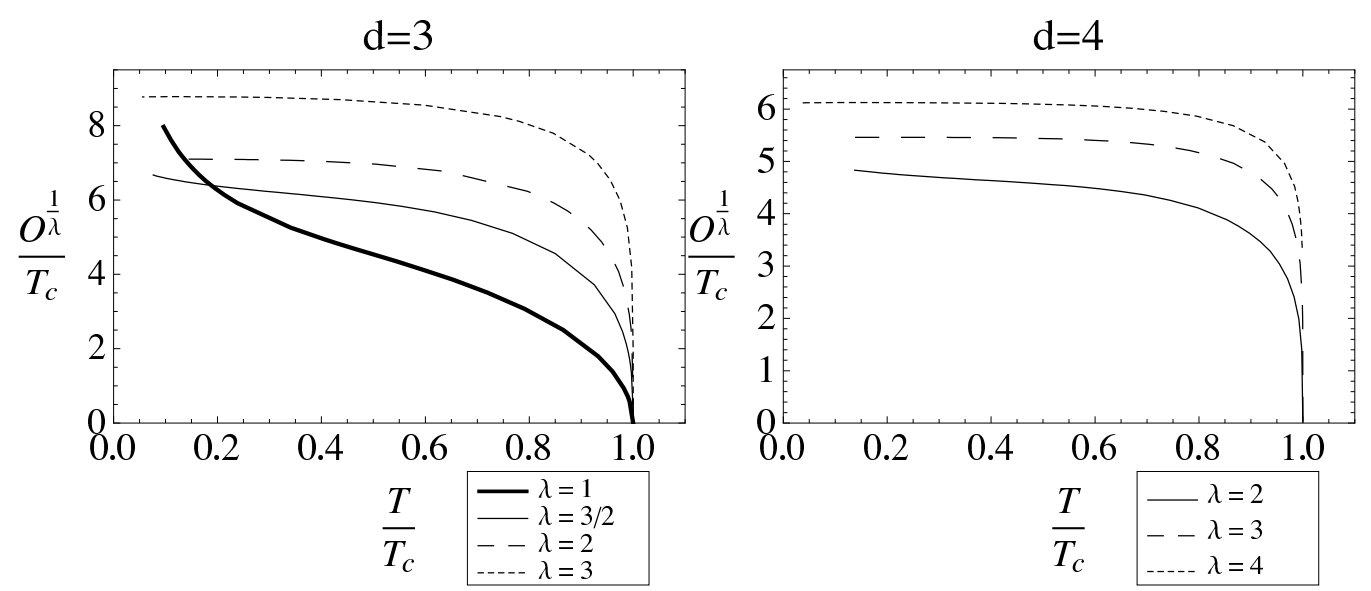

Figure 3: The energy gap $v s$. temperature for various scaling dimensions at $d=3$ (left panel) and $d=4$ (right panel [4].

The behavior of the energy is shown in fig. 3, obtained numerically for $d=3,4$ [ [4]. For scaling dimensions $\Delta>\frac{d}{2}$ (above the BF bound), the energy gap converges at zero temperature, whereas for $\Delta<\frac{d}{2}$, it diverges. The behavior cannot be conclusively determined from the numerical graph at the BF bound $\left(\Delta=\frac{d}{2}\right)$. I shall show analytically that it diverges, albeit very mildly.

In the probe limit, we have to consider the system of zeroth-order equations,

$$
\begin{aligned}
-F_{0}^{\prime \prime}+\frac{1}{z}\left[\frac{d}{1-z^{d}}-1-2 \Delta\right] F_{0}^{\prime}+\frac{\Delta^{2} z^{d-2}}{1-z^{d}} F_{0}-\frac{1}{\left(1-z^{d}\right)^{2}} \Phi_{0}^{2} F_{0} & =0 \\
\Phi_{0}^{\prime \prime}-\frac{d-3}{z} \Phi_{0}^{\prime}-\frac{b^{2 \Delta} z^{2(\Delta-1)}}{1-z^{d}} F_{0}^{2} \Phi_{0} & =0
\end{aligned}
$$


The scalar field $F_{0}(z)$ has a smooth limit as $T \rightarrow 0$ for $\Delta=\Delta_{-} \leq \frac{d}{2}$,

$$
F_{0}(z)=\frac{\Gamma^{2}\left(1-\frac{\Delta}{d}\right)}{\Gamma\left(1-\frac{2 \Delta}{d}\right)} F\left(\frac{\Delta}{d}, \frac{\Delta}{d} ; 1 ; 1-z^{d}\right)
$$

This is not the case for $\Delta=\Delta_{+}>\frac{d}{2}$ and needs to be considered separately.

The field equations can be solved by iteration at low temperatures ( $b \rightarrow \infty$ limit),

$$
\begin{aligned}
-F_{0}^{(n+1)^{\prime \prime}}+\frac{1}{z}\left[\frac{d}{1-z^{d}}-1-2 \Delta\right] F_{0}^{(n+1)^{\prime}}+\frac{\Delta^{2} z^{d-2}}{1-z^{d}} F_{0}^{(n+1)} & =\frac{\mu^{2}}{\left(1-z^{d}\right)^{2}}\left[\hat{\Phi}_{0}^{(n+1)}\right]^{2} F_{0}^{(n)} \\
\hat{\Phi}_{0}^{(n+1) \prime \prime}-\frac{d-3}{z} \hat{\Phi}_{0}^{(n+1) \prime}-\frac{b^{2 \Delta} z^{2(\Delta-1)}}{1-z^{d}}\left[F_{0}^{(n)}\right]^{2} \hat{\Phi}_{0}^{(n+1)} & =0
\end{aligned}
$$

starting with

$$
F_{0}^{(0)}(z)=1, \quad \hat{\Phi}_{0}^{(0)}(z)=0
$$

I defined

$$
\Phi_{0}(z)=\mu \hat{\Phi}_{0}(z), \quad \hat{\Phi}_{0}(0)=1,
$$

where $\mu$ is the chemical potential.

At the $n$th step, one obtains for the scalar field

$$
\begin{aligned}
F_{0}^{(n+1)}(z)= & \mathscr{F}_{1}(z)\left[1+\mu^{2} \int_{0}^{z} \frac{d z^{\prime}}{1-\left(z^{\prime}\right)^{d}}\left(z^{\prime}\right)^{2 \Delta+1-d} \mathscr{F}_{2}\left(z^{\prime}\right)\left[\hat{\Phi}_{0}^{(n+1)}\left(z^{\prime}\right)\right]^{2} F_{0}^{(n)}\left(z^{\prime}\right)\right] \\
& -\mathscr{F}_{2}(z) \mu^{2} \int_{0}^{z} \frac{d z^{\prime}}{1-\left(z^{\prime}\right)^{d}}\left(z^{\prime}\right)^{2 \Delta+1-d} \mathscr{F}_{1}\left(z^{\prime}\right)\left[\hat{\Phi}_{0}^{(n+1)}\left(z^{\prime}\right)\right]^{2} F_{0}^{(n)}\left(z^{\prime}\right)
\end{aligned}
$$

where

$$
\mathscr{F}_{1}(z)=F\left(\frac{\Delta}{d}, \frac{\Delta}{d} ; \frac{2 \Delta}{d} ; z^{d}\right), \mathscr{F}_{2}(z)=\frac{z^{d-2 \Delta}}{d-2 \Delta} F\left(1-\frac{\Delta}{d}, 1-\frac{\Delta}{d} ; 2-\frac{2 \Delta}{d} ; z^{d}\right)
$$

with the boundary condition $F_{0}^{(n+1)}(0)=1$. At the horizon, this function diverges. Demanding regularity at $z=1$ fixes $\mu$.

For $n=0$, one obtains for the electrostatic potential

$$
\hat{\Phi}_{0}^{(1)}(z)=\frac{2}{\Gamma(v)(2 \Delta)^{v}}(b z)^{\frac{d-2}{2}}\left[K_{v}\left(\frac{(b z)^{\Delta}}{\Delta}\right)-\frac{K_{v}\left(\frac{b^{\Delta}}{\Delta}\right)}{I_{v}\left(\frac{b^{\Delta}}{\Delta}\right)} I_{v}\left(\frac{(b z)^{\Delta}}{\Delta}\right)\right], \quad v=\frac{d-2}{2 \Delta},
$$

in terms of Bessel functions. The second Bessel function has an exponentially small coefficient, $\mathscr{O}\left(\sim e^{-2 b^{\Delta} / \Delta}\right)$, and can be neglected at low $T$.

The charge density is

$$
\frac{\rho_{0}}{b^{d-2}}=-\frac{\mu}{(2 \Delta)^{2 v}}
$$

For the scalar field one obtains

$$
\begin{aligned}
F_{0}^{(1)}(z)= & \mathscr{F}_{1}(z)\left[1+\mu^{2} \int_{0}^{z} \frac{d z^{\prime}}{1-\left(z^{\prime}\right)^{d}}\left(z^{\prime}\right)^{2 \Delta+1-d} \mathscr{F}_{2}\left(z^{\prime}\right)\left[\hat{\Phi}_{0}^{(1)}\left(z^{\prime}\right)\right]^{2}\right] \\
& -\mathscr{F}_{2}(z) \mu^{2} \int_{0}^{z} \frac{d z^{\prime}}{1-\left(z^{\prime}\right)^{d}}\left(z^{\prime}\right)^{2 \Delta+1-d} \mathscr{F}_{1}\left(z^{\prime}\right)\left[\hat{\Phi}_{0}^{(1)}\left(z^{\prime}\right)\right]^{2}
\end{aligned}
$$


which has a logarithmic singularity at the horizon,

$$
F_{0}^{(1)}(z) \approx-\left[\frac{\Gamma\left(\frac{2 \Delta}{d}\right)}{\Gamma^{2}\left(\frac{\Delta}{d}\right)}\left(1+\mu^{2} a_{2}\right)-\frac{\Gamma\left(2-\frac{2 \Delta}{d}\right)}{(d-2 \Delta) \Gamma^{2}\left(1-\frac{\Delta}{d}\right)} \mu^{2} a_{1}\right] \ln (1-z)
$$

Demanding regularity at the horizon, the coefficient of the logarithm must vanish, which fixes the chemical potential,

$$
\frac{1}{\mu^{2}}=\frac{\Gamma\left(2-\frac{2 \Delta}{d}\right) \Gamma^{2}\left(\frac{\Delta}{d}\right)}{(d-2 \Delta) \Gamma\left(\frac{2 \Delta}{d}\right) \Gamma^{2}\left(1-\frac{\Delta}{d}\right)} a_{1}-a_{2} .
$$

Explicitly,

$$
a_{1}=\frac{1}{b^{2 \Delta+2-d}} \frac{(d-2) \Gamma(1-v)}{(2 \Delta)^{2 v} \Gamma(v)}+\ldots, a_{2}=\frac{1}{b^{2}} \frac{\sqrt{\pi} \Delta^{\frac{2}{\Delta}-1} \Gamma\left(\frac{1}{\Delta}\right) \Gamma\left(\frac{d-1}{\Delta}\right) \Gamma\left(\frac{d}{2 \Delta}\right)}{(d-2 \Delta) \Gamma^{2}(v) 2^{2 v} \Gamma\left(\frac{d+\Delta}{2 \Delta}\right)}+\ldots
$$

Evidently, for $\Delta<\frac{d}{2}, a_{2} / a_{1} \rightarrow 0$ as $b \rightarrow \infty$, therefore

$$
\mu^{2} \approx \mathscr{C} b^{2 \Delta+2-d}, \mathscr{C}=\frac{(d-2 \Delta)(2 \Delta)^{2 v} \Gamma(v) \Gamma\left(\frac{2 \Delta}{d}\right) \Gamma^{2}\left(1-\frac{\Delta}{d}\right)}{(d-2) \Gamma(1-v) \Gamma\left(2-\frac{2 \Delta}{d}\right) \Gamma^{2}\left(\frac{\Delta}{d}\right)}
$$

It is easily seen (using standard hypergeometric identities) that the low temperature expression (3.11), with the choice (3.13) of $\mu$, reduces to the $T=0$ expression (3.3) as $b \rightarrow \infty$. In fig. 5, the analytic expression (3.11) is compared with numerical results at temperature $T / T_{c} \approx 0.1$ in $d=3$ dimensions. The agreement is excellent, as the corresponding curves are almost indistinguishable. A similar comparison in $d=4$ dimensions is shown in fig. 6. Higher-order corretions are less than $1 \%$, as seen in fig. 7. showing that the iterative procedure for the analytic solution converges rapidly at low temperatures.

For the charge density, we deduce from (3.10) and 3.15),

$$
\rho_{0} \sim b^{\frac{d}{2}+\Delta-1} .
$$

Using

$$
\frac{\left\langle\mathscr{O}_{\Delta}\right\rangle^{1 / \Delta}}{T_{c}} \sim b \rho_{0}^{-\frac{1}{d-1}}, \frac{T}{T_{c}} \sim \rho_{0}^{-\frac{1}{d-1}},
$$

we finally obtain for the energy gap

$$
\frac{E_{g}}{T_{c}}=\frac{\left\langle\mathscr{O}_{\Delta}\right\rangle^{1 / \Delta}}{T_{c}}=\gamma\left(\frac{T}{T_{c}}\right)^{-\frac{d / 2-\Delta}{d / 2+\Delta-1}}
$$

showing that the condensate diverges as $T \rightarrow 0$. Notice that the exponent depends on the dimensions of both the operator and spacetime. The constant of proportionality $\gamma$ can be found analytically and is plotted in fig. 4 . As the scaling dimension approaches the BF bound $\left(\Delta \rightarrow \frac{d}{2}\right)$, we have $\gamma \rightarrow 0$, indicating that the power law behavior changes. Thus, at the BF bound, $\Delta=\frac{d}{2}$, one needs to exercise care. Letting $\Delta=\frac{d}{2}-\varepsilon$,

$$
\frac{1}{\mu^{2}}=\frac{(d-2) \Gamma\left(\frac{2}{d}\right)}{d^{2\left(1-\frac{2}{d}\right)} \Gamma\left(1-\frac{2}{d}\right)}\left[\frac{1}{2 \varepsilon b^{2-2 \varepsilon}}-\frac{1}{2 \varepsilon b^{2}}+\ldots\right]
$$




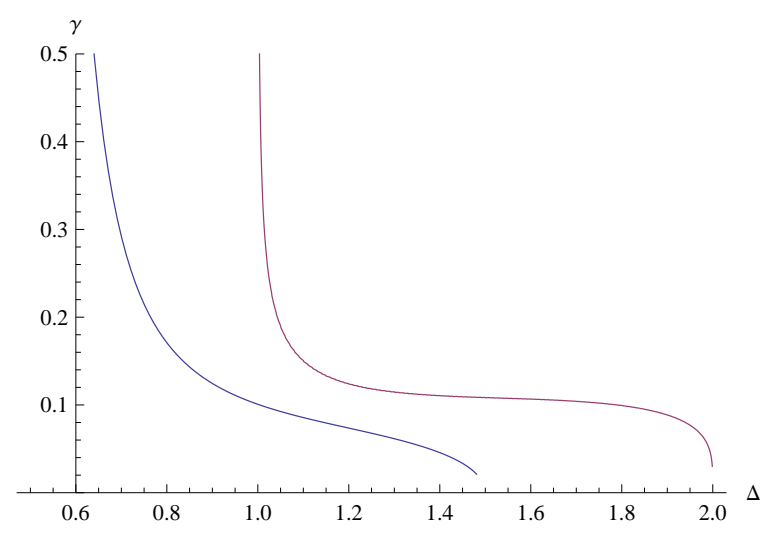

Figure 4: The parameter $\gamma$ in the energy gap $v s \Delta$. Curve on left (right) is for $d=3(d=4)$ [7].
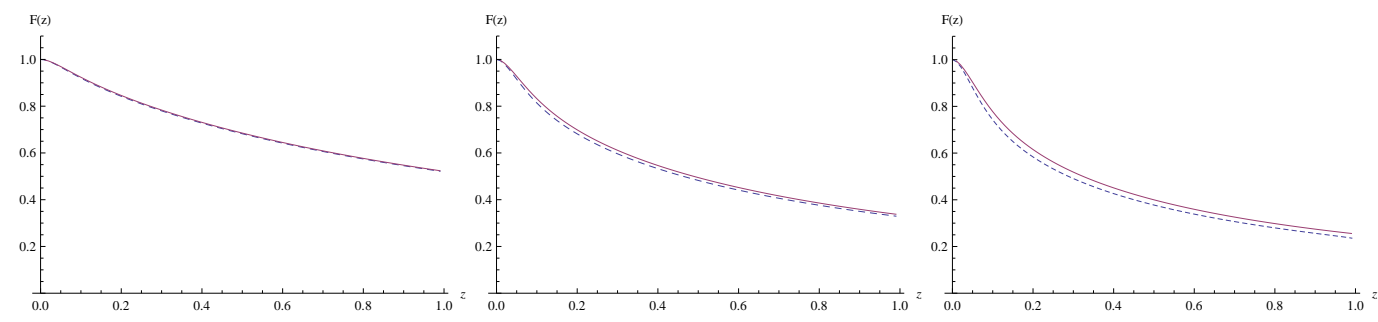

Figure 5: The field $F$ for $\Delta=1.2$ (left panel), 1.4 (middle panel), 1.5 (right panel) and $d=3$. First-order analytic results (dashed lines) compared with exact numerical results (almost indistinguishable solid lines) at $T / T_{c} \approx 0.1$ 㠴.

and taking the limit $\varepsilon \rightarrow 0$, one deduces at the BF bound

$$
\frac{\mu^{2}}{b^{2}}=\frac{d^{2\left(1-\frac{2}{d}\right)} \Gamma\left(1-\frac{2}{d}\right)}{(d-2) \Gamma\left(\frac{2}{d}\right)\left[\ln b+\beta_{d}+o\left(b^{0}\right)\right]}
$$

where $\beta_{d}$ is a constant that depends on the dimension and is easily computed (e.g., for $d=3$, $\beta_{3} \approx 1.75$ ).

The charge density at the BF bound behaves as

$$
\rho_{0} \sim b^{d-1}(\ln b)^{-1 / 2}
$$

and the energy gap as

$$
\frac{E_{g}}{T_{c}}=\frac{\left\langle\mathscr{O}_{\Delta}\right\rangle^{1 / \Delta}}{T_{c}} \sim(\ln b)^{\frac{1}{2(d-1)}} \sim\left(\ln \frac{T_{c}}{T}\right)^{\frac{1}{2(d-1)}}
$$

showing that the energy gap diverges at the BF bound, albeit very mildly. This mild divergence was missed in earlier numerical studies (see fig. 3).

Above the BF bound $(\Delta>d / 2)$, as $T \rightarrow 0$, we have $F_{0} \approx 1$ near the boundary $(z \lesssim 1 / b)$, but asymptotically (large $z \gtrsim 1 / b), F_{0} \sim(b z)^{d-2 \Delta}$, which does not have a smooth limit as $T \rightarrow 0$. Therefore, one cannot apply perturbation theory. Instead, one can approximate $F_{0}$ by

$$
F_{0}(z)=\left\{\begin{array}{cc}
1 & , z \leq \alpha / b \\
\left(\frac{b z}{\alpha}\right)^{d-2 \Delta} & , z>\alpha / b
\end{array}\right.
$$



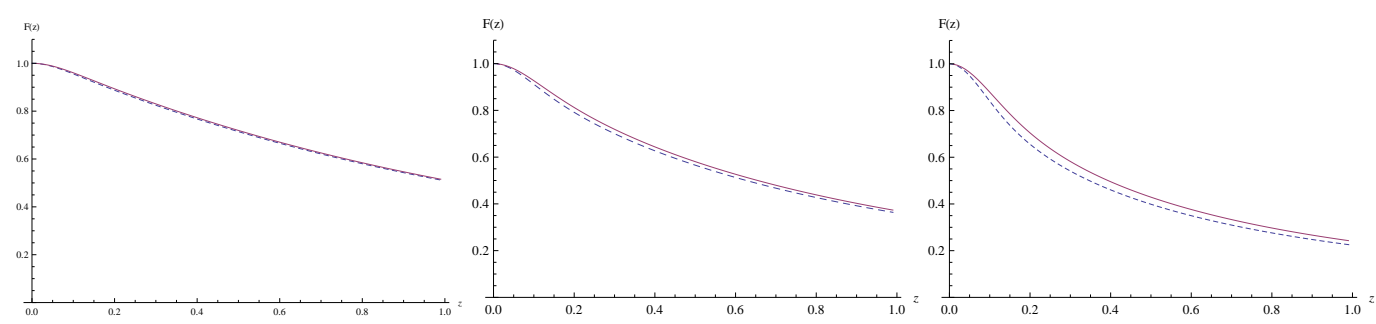

Figure 6: The field $F$ for $\Delta=1.6$ (left panel), 1.8 (middle panel), 2 (right panel) and $d=4$. Upper two curves are from first-order analytic expression and exact numerical results (almost indistinguishable) at $T / T_{c} \approx 0.2$; lower dashed curves are the $T=0$ limit 沛].
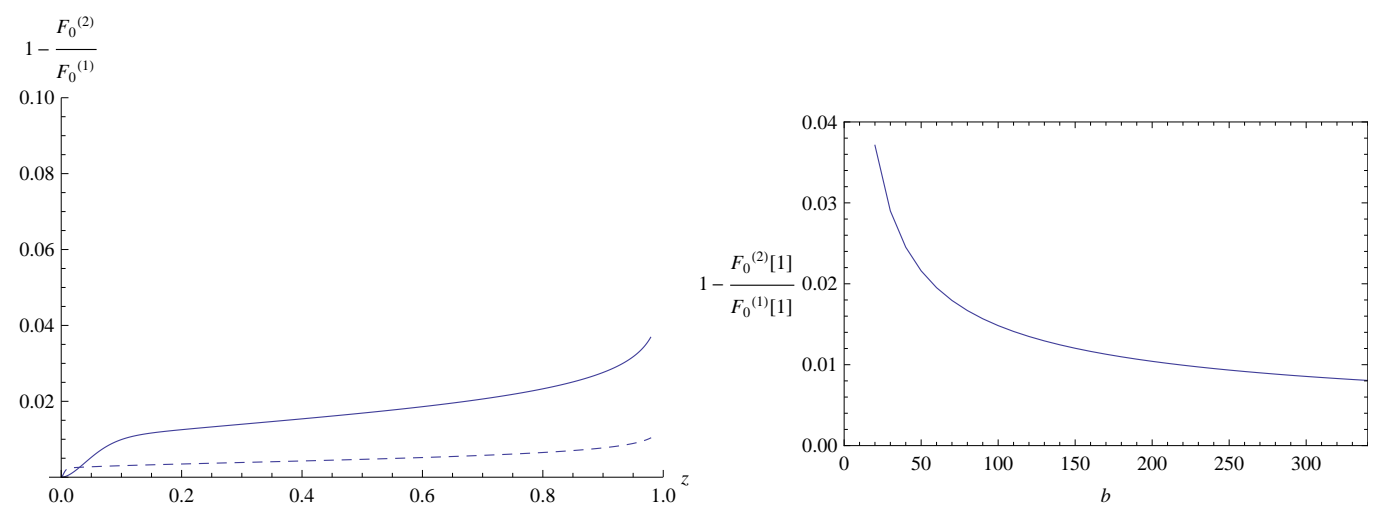

Figure 7: Corrections are $\lesssim 1 \%$ for $T / T_{c} \lesssim 0.1$ (left panel) and vanish as $T \rightarrow 0$ (right panel) [㠼.

and find $\alpha$ by a variational method [6]. One can then find an analytic estimate of the energy gap which remains finite as $T \rightarrow 0$.

Since for $\Delta<d / 2$ the energy gap diverges as $T \rightarrow 0$, it is necessary to include corrections and move away from the probe limit $(q \rightarrow \infty)$ to obtain a physical system at low temperatures. At first order, we obtain for the functions determining the metric,

$$
\begin{aligned}
z f_{1}^{\prime}-d f_{1} & =\frac{(b z)^{2 \Delta}}{4(d-1)}\left[\left(m^{2}+\Delta^{2} f_{0}+\frac{z^{2} \Phi_{0}^{2}}{(b z)^{2 \Delta}}\right) F_{0}^{2}+2 \Delta z f_{0} F_{0} F_{0}^{\prime}+z^{2} f_{0}{F_{0}^{\prime}}^{2}+\frac{z^{4}}{(b z)^{2 \Delta}} \Phi_{0}^{\prime 2}\right] \\
z \chi_{1}^{\prime} & =\frac{(b z)^{2 \Delta}}{d-1}\left[\left(\Delta^{2}+\frac{z^{2} \Phi_{0}^{2}}{f_{0}^{2}}\right) F_{0}^{2}+2 \Delta z F_{0} F_{0}^{\prime}+z^{2} F_{0}^{\prime 2}\right]
\end{aligned}
$$

The solution is

$$
f_{1}(z)=-\frac{\Delta}{4(d-1)}(b z)^{2 \Delta}\left[2-z^{d}-z^{d-2 \Delta}\right]+\ldots, \quad \chi_{1}(z)=-\frac{\Delta}{2(d-1)}(b z)^{2 \Delta}+\ldots
$$

Similar results are also easily obtained for the first-order corrections to the scalar and electrostatic potential ( $\Psi_{1}$ and $\Phi_{1}$, respectively). In particular, for the temperature, we deduce the first-order expression

$$
\frac{T}{(q \rho)^{1 /(d-1)}}=\frac{d}{4 \pi \rho_{0}^{1 /(d-1)}}\left[1+\lambda \frac{b^{2 \Delta}}{q^{2}}+\ldots\right]
$$

where $\lambda>0$ (the temperature receives a positive correction away from the probe limit) and can be found explicitly. It is now clear why the probe limit fails as $T \rightarrow 0$. For the expansion in $1 / q^{2}$ to 
be valid, we ought to have

$$
b \lesssim q^{1 / \Delta}
$$

For a given $q$, this places a lower bound on the temperature above which the probe limit is valid. While $T=0$ is unattainable for finite $q$, the temperature below which the probe limit is not valid can be made arbitrarily low by choosing a sufficiently large $q$. Thus, even though the probe limit $(q \rightarrow \infty)$ is not always a physical system, its properties are a good approximation to corresponding properties of physical systems (of finite $q$ ). This approximation becomes better with increasing $q$ and the $1 / q^{2}$ expansion is valid over a wider range of temperatures.

\section{Conductivity in the probe limit}

I am interested in calculating the conductivity analytically at low temperatures. I shall obtain the conductivity $\sigma$ as a function of the rescaled frequency

$$
\hat{\omega}=\frac{\omega}{b}=\frac{\omega}{\left\langle\mathscr{O}_{\Delta}\right\rangle^{1 / \Delta}}
$$

The function $\sigma(\hat{\omega})$ has a well-defined limit as $q \rightarrow \infty$ (probe limit) down to zero temperature even though the condensate $\left\langle\mathscr{O}_{\Delta}\right\rangle$ diverges. As I discussed above, the probe limit, which is not a physical state at low temperatures, can be arbitrarily well approximated by physical states of sufficiently large $q$. The conductivity of these states can be obtained as a $1 / q^{2}$ expansion with the conductivity in the probe limit serving as the zeroth order term in the expansion.

For definiteness, let $d=3$. The case $d=4$ will be discussed later.

The conductivity on the AdS boundary is found by applying a sinusoidal electromagnetic perturbation in the bulk of frequency $\omega$ obeying the wave equation

$$
-\frac{d^{2} A}{d r_{*}^{2}}+V A=\omega^{2} A, \quad V=\frac{2 q^{2}}{z^{2}} f \Psi^{2}
$$

where $A$ is any component of the perturbing electromagnetic potential along the boundary, subject to ingoing boundary condition at the horizon,

$$
A \sim e^{-i \omega r_{*}} \sim(1-z)^{-i \omega / 3}
$$

as $z \rightarrow 1\left(r_{*} \rightarrow-\infty\right)$, where $r_{*}$ is the tortoise coordinate

$$
r_{*}=\int \frac{d z}{f(z)}=\frac{1}{6}\left[\ln \frac{(1-z)^{3}}{1-z^{3}}-2 \sqrt{3} \tan ^{-1} \frac{\sqrt{3} z}{2+z}\right]
$$

with the integration constant chosen so that the boundary is at $r_{*}=0$.

The wave equation reads

$$
\frac{d}{d z}\left[\left(1-z^{3}\right) \frac{d A}{d z}\right]-\left[b^{2 \Delta} z^{2 \Delta-2} F^{2}(z)-\frac{\omega^{2}}{1-z^{3}}\right] A=0
$$

Set

$$
A=(1-z)^{-i \omega / 3} e^{-i \omega z / 3} \mathscr{A}(z)
$$


where I included a convenient factor of $e^{-i \omega z / 3}$ so that only $\mathscr{A}(z)$ contributes to the conductivity. The wave equation becomes

$$
\begin{aligned}
& -3\left(1-z^{3}\right) \mathscr{A}^{\prime \prime}+z\left[9 z-2\left(1+z+z^{2}\right) i \omega\right] \mathscr{A}^{\prime} \\
& +\left[3 b^{2 \Delta} z^{2 \Delta-2} F^{2}(z)-\left(1+2 z+3 z^{2}\right) i \omega-\frac{\left(3+2 z+z^{2}\right)\left(3+z+z^{2}+z^{3}\right)}{3\left(1+z+z^{2}\right)} \omega^{2}\right] \mathscr{A}=0
\end{aligned}
$$

Regularity of $\mathscr{A}$ at the horizon $(z=1)$ implies the boundary condition

$$
(3-2 i \omega) \mathscr{A}^{\prime}(1)+\left(b^{2 \Delta} F^{2}(1)-2 i \omega-\frac{4 \omega^{2}}{3}\right) \mathscr{A}(1)=0
$$

In the low temperature limit (large $b$ ), it is convenient to rescale $z \rightarrow z / b$, and solve as a series expansion in $1 / q^{2}$.

The zeroth order term (probe limit) is obtained by replacing $F$ by $F_{0}$, for $\Delta \leq \frac{3}{2}$ (so that $F_{0}$ has a smooth zero temperature limit),

$$
-\mathscr{A}^{\prime \prime}+\left[z^{2 \Delta-2}-\hat{\omega}^{2}\right] \mathscr{A}=0
$$

where I used $F(z / b) \rightarrow F(0)=1$, for large $b$. For $1<\Delta \leq \frac{3}{2}$, there are two linearly independent solutions, $\mathscr{A}_{ \pm}$, distinguished by their asymptotic behavior,

$$
\mathscr{A}_{ \pm} \sim e^{ \pm \frac{1}{\Delta} z^{\Delta}}, \quad z \rightarrow \infty
$$

The general solution can be written as a linear combination,

$$
\mathscr{A}=c^{+} \mathscr{A}_{+}+c^{-} \mathscr{A}_{-}
$$

Applying the boundary condition (4.8) at horizon, we deduce

$$
\frac{c^{+}}{c^{-}} \sim e^{-\frac{2}{\Delta} b^{\Delta}}
$$

so at low temperatures,

$$
c^{+} \ll c^{-}
$$

i.e., $\mathscr{A} \rightarrow 0$ as $z \rightarrow \infty$.

For scaling dimension $\Delta=1$, one obtains the exact solution

$$
\mathscr{A}(z)=e^{-\sqrt{1-\hat{\omega}^{2}} b z}
$$

(whereas $\mathscr{A}_{+}(z)=e^{+\sqrt{1-\hat{\omega}^{2}} b z}$ ) with arbitrary normalization, where I restored the scaling parameter $b$.

Thus, the low temperature limit of the conductivity is

$$
\sigma(\hat{\omega})=\frac{i}{\hat{\omega}} \sqrt{1-\hat{\omega}^{2}}
$$

Notice that $\Re \sigma=0$ for $\hat{\omega} \leq 1$, therefore the gap is

$$
\omega_{g}=\left\langle\mathscr{O}_{1}\right\rangle
$$


At low $T$, corrections are $\sim \mathscr{O}\left(e^{-2 b}\right)$, therefore

$$
\Re \sigma \sim e^{-E_{g} / T}, \quad E_{g}=\frac{3\left\langle\mathscr{O}_{1}\right\rangle}{2 \pi} \approx 0.48 \omega_{g},
$$

to be compared with the BCS result for the energy gap $E_{g}=\omega_{g} / 2=0.50 \omega_{g}$.

For scaling dimension $\Delta=\frac{3}{2}$ (BF bound),

$$
\mathscr{A}(z)=\mathscr{A}_{-}(z)=\mathrm{Ai}\left(b z-\hat{\omega}^{2}\right),
$$

whereas $\mathscr{A}_{+}(z)=\operatorname{Bi}\left(b z-\hat{\omega}^{2}\right)$.

At $T=0$ in the probe limit, the quasinormal frequencies have moved to the real axis yielding an infinite set of normal frequencies which are solutions of

$$
\operatorname{Ai}\left(-\hat{\omega}^{2}\right)=0
$$

giving rise to an infinite tower of real frequencies determined by the zeroes of the Airy function.

The $T=0$ conductivity in the probe limit is

$$
\sigma(\hat{\omega})=\frac{i}{\hat{\omega}} \frac{\operatorname{Ai}^{\prime}\left(-\hat{\omega}^{2}\right)}{\operatorname{Ai}\left(-\hat{\omega}^{2}\right)}
$$

The normal modes are the poles of the conductivity. At zero temperature, $\Re \sigma=0$, except at the poles of $\mathfrak{I} \sigma$ where $\mathfrak{R} \sigma$ diverges as a $\delta$-function. The gap is

$$
\omega_{g} \approx 1.5\left\langle\mathscr{O}_{3 / 2}\right\rangle^{2 / 3}
$$

At low temperatures,

$$
\Re \sigma \sim e^{-\frac{4}{3} b^{3 / 2}}=e^{-\left(E_{g} / T\right)^{3 / 2}}, \quad E_{g}=\frac{1}{\pi}\left(\frac{3}{4}\right)^{1 / 3}\left\langle\mathscr{O}_{3 / 2}\right\rangle^{2 / 3} \approx 0.2 \omega_{g}
$$

to be compared with the BCS result $E_{g}=0.5 \omega_{g}$.

However, the system at $T=0$ is not physical in the probe limit, so there is no physical conductivity that has the poles found above. For a physical low temperature system, we need to include $1 / q^{2}$ corrections. These shift the poles off the real axis resulting in spikes for the conductivity of a physical system.

To see this explicitly, consider the first-order correction $\delta \mathscr{A}$ which obeys the wave equation

$$
-\delta \mathscr{A}^{\prime \prime}+\left[z-\hat{\omega}^{2}\right] \boldsymbol{\delta} \mathscr{A}=-\frac{1}{3\left(1-z^{3}\right)} \mathscr{H}_{1} \mathscr{A}
$$

where

$$
\begin{aligned}
\mathscr{H}_{1}= & z\left[9 z-2\left(1+z+z^{2}\right) i \omega\right] \frac{d}{d z}+3 b^{3} z\left(2 F_{1}(z)+z^{3}\right)-\left(1+2 z+3 z^{2}\right) i \omega \\
& +\frac{z^{2}\left(1-15 z-12 z^{2}-10 z^{3}\right)}{3\left(1+z+z^{2}\right)} \omega^{2}
\end{aligned}
$$




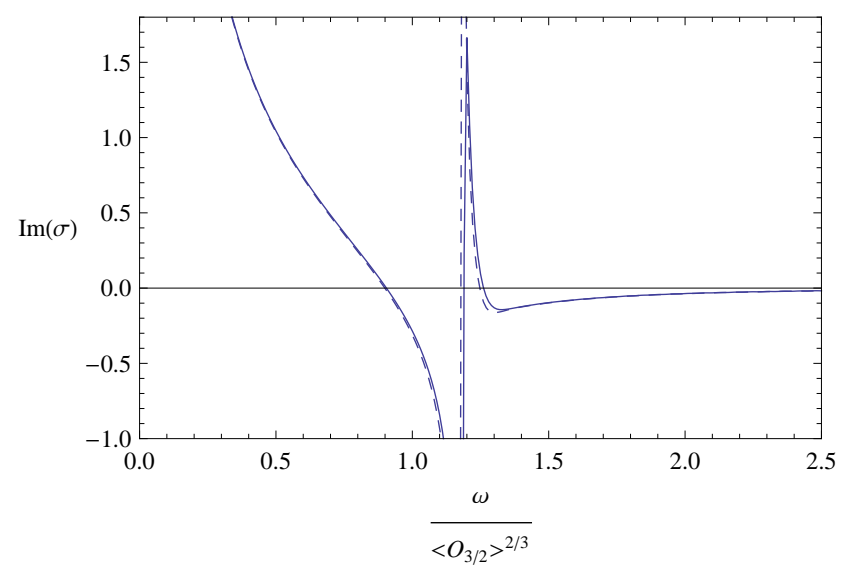

Figure 8: The imaginary part of the conductivity in $d=3$ using the analytic expression for the scalar field (dotted line) compared with the exact numerical solution (solid line) at $\frac{T}{T_{C}} \approx .1$ 㠴.
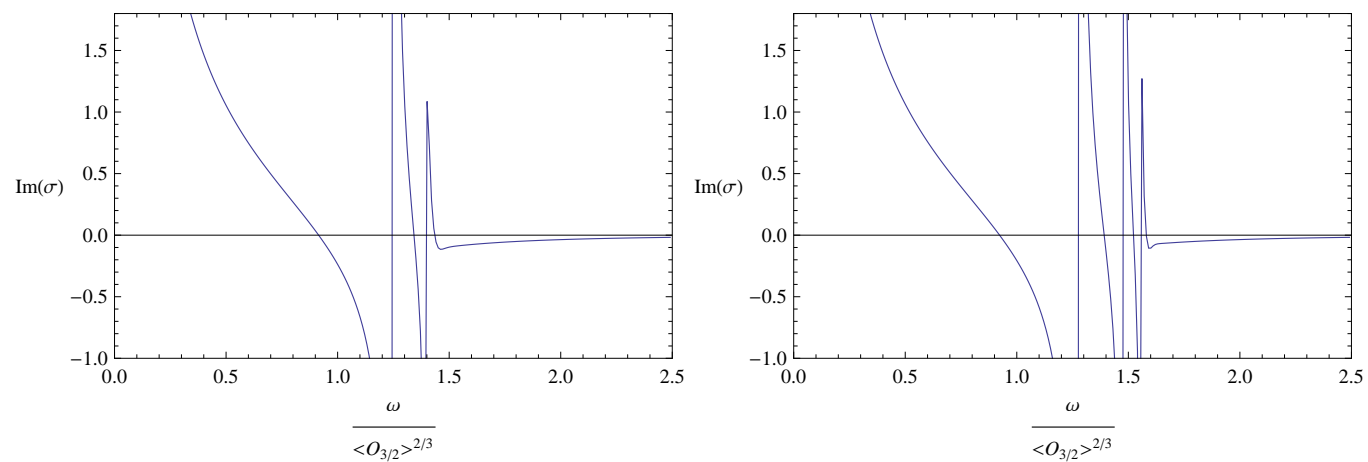

Figure 9: The imaginary part of the conductivity $v s$. frequency in $d=3$ using the analytic expression for $F$ at $\frac{T}{T_{c}} \approx .05$ (left), .04 (right). As the temperature decreases, poles move on to the real axis [7].

The real frequencies found above get shifted away from the real axis,

$$
\delta \hat{\omega}=\frac{\pi \operatorname{Bi}\left(-\hat{\omega}^{2}\right)}{3 \hat{\omega} \mathrm{Ai}^{\prime}\left(-\hat{\omega}^{2}\right)} \int_{0}^{1} \frac{d z}{1-z^{3}} \operatorname{Ai}\left(b z-\hat{\omega}^{2}\right) \mathscr{H}_{1} \operatorname{Ai}\left(b z-\hat{\omega}^{2}\right)
$$

This expression is valid for low frequencies and low temperatures. As we heat up the system, most modes disappear and we are left with a finite number of quasinormal modes. Their number decreases as we increase the temperature. Conversely, as we cool down the system, modes shift toward the real axis, and we obtain an increasing number of spikes in the conductivity.

Figure 8 depicts the imaginary part of the conductivity at temperature $T / T_{c} \approx .1$ in comparison with the exact numerical solution. The agreement is very good even at such high temperature at which only one quasinormal mode is left. Unfortunately, this is the low temperature limit attained by numerical analysis as numerical instabilities prohibit one from lowering the temperature further. Using the above analytical results, one can see in figure 9 the emergence of an increasing number of poles as one lowers the temperature to $T / T_{c} \approx .06$ and .04 . Finally in figure 10, the lower temperature $\left(T / T_{c} \approx .01\right)$ result is compared with the zero temperature analytic expression $(4.20)$ demonstrating convergence. 


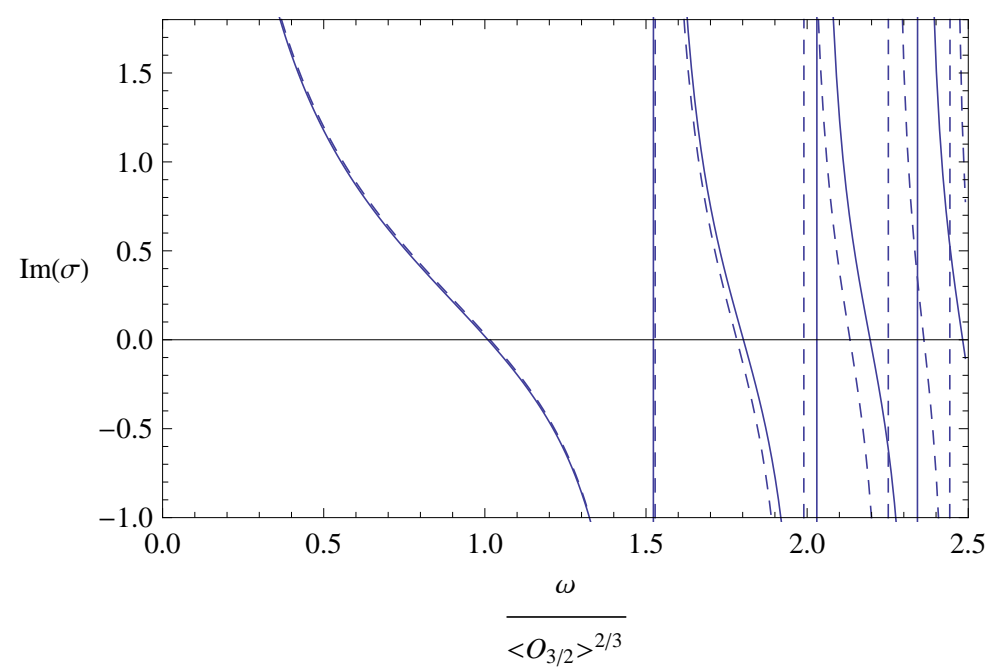

Figure 10: Comparison of the imaginary part of the conductivity in $d=3$ using the analytic expression for $\mathrm{F}$ at $\frac{T}{T_{c}} \approx .01$ (dotted line) and the zero temperature limit (solid line) [7].

For $\Delta>3 / 2$, the wave equation (4.9) is not valid because the scalar does not have a smooth zero temperature limit. On the other hand the probe limit is physical down to zero temperatures. The potential

$$
V=b^{2 \Delta} z^{2 \Delta-2}\left(1-z^{3}\right) F(b z)
$$

attains a maximum of order $b^{2(2-\Delta)}$ for $\Delta<2$. Therefore, at zero temperature it has infinite height. However, the width becomes infinitely narrow leading to a finite tower of poles for the conductivity (quasinormal modes). In the zero temperature limit, the number of modes increases as one approaches the BF bound and decreases away from it.

Similar results hold for $d=4$. At the BF bound, $\Delta=2$, in the probe limit, the wave equation reads

$$
A^{\prime \prime}-\frac{1}{z} A^{\prime}-\left[b^{4} z^{2}-\omega^{2}\right] A=0,
$$

whose acceptable solution can be written in terms of a Whittaker function,

$$
A=W_{\frac{\hat{\omega}^{2}}{4}, \frac{1}{2}}\left(b^{2} z^{2}\right)
$$

(The other solution diverges as $z \rightarrow \infty$.) At the boundary $(z \rightarrow 0)$, it has a logarithmic divergence which we need to subtract before we can calculate quasi-normal modes and the conductivity [ $₫$. The conductivity is then given by

$$
\sigma(\hat{\omega})=\frac{2}{i \hat{\omega}} \frac{A_{2}}{A_{0}}+\frac{i \hat{\omega}}{2}
$$

with an arbitrarily chosen cutoff, where

$$
A(z)=A_{0}+A_{2} b^{2} z^{2}-A_{0} \frac{\hat{\omega}^{2}}{2} b^{2} z^{2} \ln \left(b^{2} z^{2}\right)+\ldots
$$

Using the expansion for small arguments,

$$
W_{\frac{\omega^{2}}{4}, \frac{1}{2}}\left(b^{2} z^{2}\right)=-\frac{2}{\hat{\omega}^{2} \Gamma\left(-\hat{\omega}^{2} / 4\right)}\left\{1-\left[1+\hat{\omega}^{2}\left(2 \gamma-1+\ln \left(b^{2} z^{2}\right)+\psi\left(1-\hat{\omega}^{2} / 4\right)\right)\right] \frac{b^{2} z^{2}}{2}\right\}+\ldots
$$




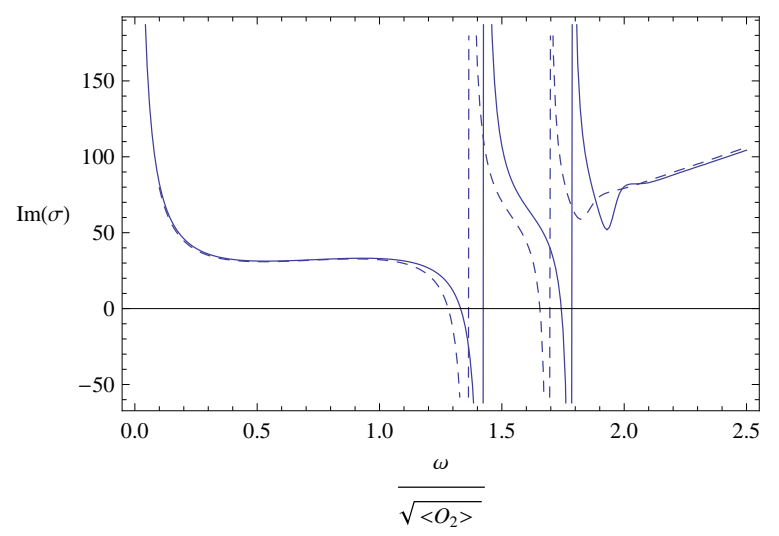

Figure 11: $\mathfrak{I} \sigma$ in $d=4$ using the analytic expression for the scalar field (dotted line) compared with the exact numerical solution (solid line) at $\frac{T}{T_{c}} \approx .17$ 沛.

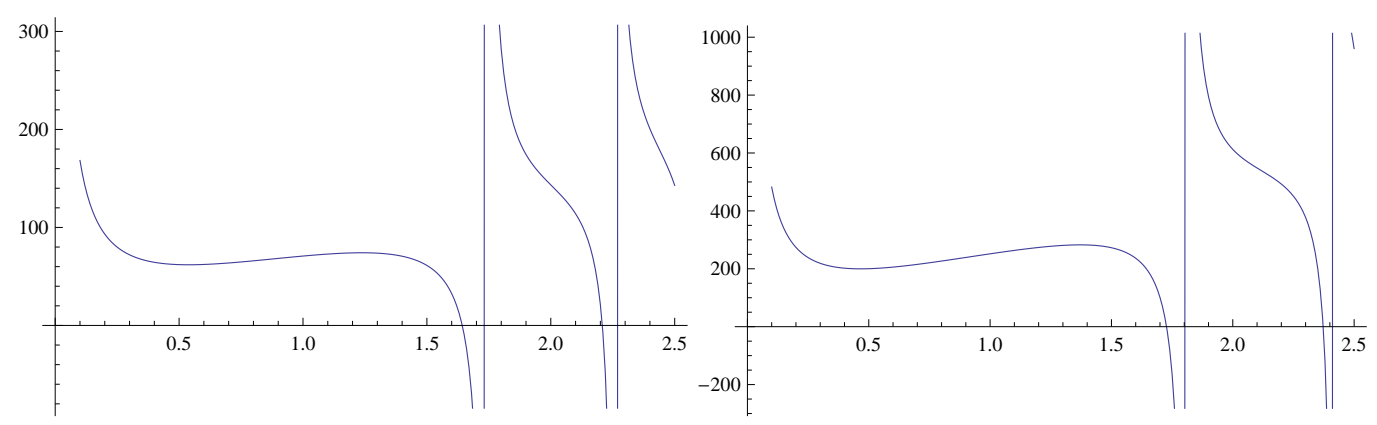

Figure 12: $\Im \sigma$ in $d=4$ using the analytic expression for $F$ at $\frac{T}{T_{c}} \approx .1$ (left), .04 (right) 吼.

we deduce the $T=0$ conductivity in the probe limit

$$
\sigma(\hat{\omega})=\frac{1}{i \hat{\omega}}+i \hat{\omega}\left[2 \gamma-\frac{1}{2}+\psi\left(1-\hat{\omega}^{2} / 4\right)\right]
$$

We have a pole at $\omega=0$, as expected and an infinite tower of real poles determined by the zeroes of the digamma (poles of the Gamma) function,

$$
\hat{\omega}=\frac{\omega_{n}}{\langle\mathscr{O}\rangle^{1 / 2}}=2 \sqrt{n}, \quad n=0,1,2, \ldots
$$

Again, these poles shift off the real axis in a physical system (quasi-normal modes) and result in spikes in the conductivity, as can be seen in figures 11 and 12. The analytic (unphysical) limit (4.33) is plotted in fig. 13.

\section{The near-extremal limit}

Next, I consider the other end of the range of $q$, i.e., small $q$. Whereas the probe limit, $q \rightarrow \infty$, corresponds to a Schwarzschild black hole, the small $q$ limit corresponds to a Reissner-Nordström black hole near extremality. On the gauge theory side of the correspondence, this translates to large chemical potential. 


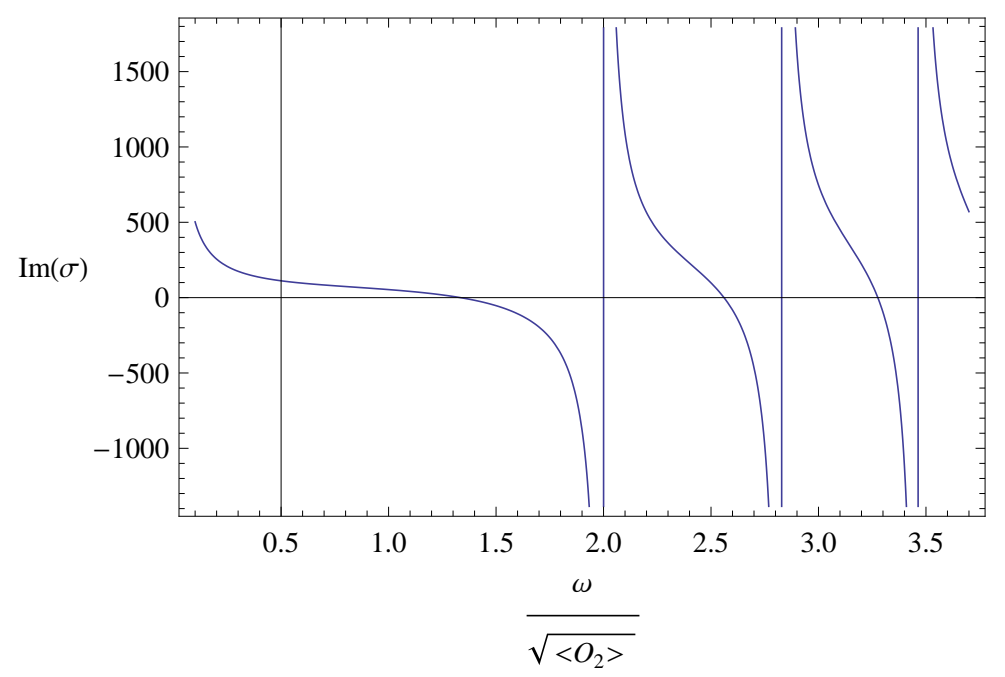

Figure 13: $\Im \sigma$ at $T=0$ in $d=4$ [沛].
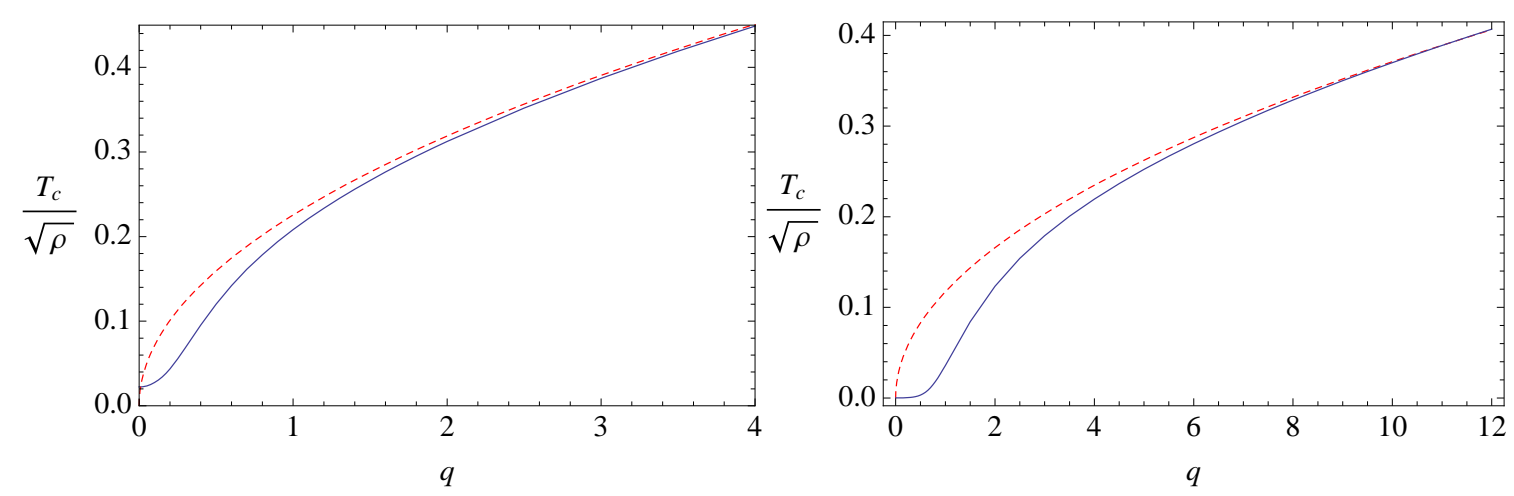

Figure 14: $\Delta=1$ (left panel), $\Delta=2$ (right panel). [12]

Numerical studies show that an instability exists even when $q=0$ (see fig. 14). It is not clear what the underlying physical mechanism is on the gauge theory (superfluid) side. Numerical analysis is cumbersome in the small $q$ limit, so to understand this regime, I shall develop analytic tools.

For definiteness, I concentrate on $d=3$ and scalar mass $m^{2}=-2$, so that the possible scaling dimensions are $\Delta=1,2$.

Above the critical temperature $\left(T \geq T_{c}\right)$, we have $\Psi=0$ and $\chi=0$. The field equations (2.4) reduce to

$$
\begin{aligned}
\Psi^{\prime \prime}+\left[\frac{f^{\prime}}{f}-\frac{2}{z}\right] \Psi^{\prime}+\left[\frac{q^{2} \Phi^{2}}{f^{2}}-\frac{m^{2}}{z^{2} f}\right] \Psi & =0 \\
\frac{z^{2}}{4} \Phi^{\prime 2}-\frac{f^{\prime}}{z}+\frac{3(f-1)}{z^{2}} & =0
\end{aligned}
$$

The first (scalar) equation has a non-zero solution only at $T=T_{c}$. The other two (Einstein-Maxwell) equations yield the RN black hole (2.10) with $d=3$, whose Hawking temperature is given by (2.11) 
with $d=3$.

To find the critical temperature $T_{c}$, we need to solve the field equation for $\Psi$.

At extremality,

$$
\frac{\rho^{2}}{4}=3, \quad T=0
$$

The general real solution to the field equation is

$\Psi=A \frac{z}{z-z_{+}}\left(\frac{z-z_{-}}{z-z_{+}}\right)^{\frac{2 \sqrt{2}-i}{2 \sqrt{3}} q}\left(\frac{1-z}{z-z_{+}}\right)^{\delta_{+}} F\left(-\delta_{-}+2 \sqrt{\frac{2}{3}} q,-\delta_{-}-\frac{i q}{\sqrt{3}} ;-2 \delta_{-} ; 2 z_{+}^{2} \frac{1-z}{z-z_{+}}\right)+$c.c.

where $z_{ \pm}$are the two complex roots of $f(z)\left(z_{-}=z_{+}^{*}\right)$, and

$$
\delta_{ \pm}=-\frac{1}{2} \pm i \frac{\sqrt{1+4 q^{2}}}{2 \sqrt{3}}
$$

are the scaling dimensions of a scalar field in $\mathrm{AdS}_{2}$ space of effective mass

$$
m_{\mathrm{eff}}^{2}=m^{2}+g^{t t} q^{2} \Phi^{2}=-2-2 q^{2}
$$

The radius of the $\mathrm{AdS}_{2}$ space is $R=1 / \sqrt{6}$.

Since both scaling dimensions are complex, neither solution is regular at the horizon. This is in accord with the fact that we are below the BF bound in $\mathrm{AdS}_{2}$ space.

Near extremality,

$$
\frac{\rho^{2}}{4}=3-4 \pi T_{c}, \quad T_{c} \ll 1
$$

The above $T=0$ result (5.3) is still approximately valid away from the horizon.

Near the horizon, perform the coordinate transformation

$$
z=1-\frac{2 \pi T_{c}}{3} \zeta
$$

The metric becomes

$$
d s^{2}=\frac{1}{6 \zeta^{2}}\left[-\left(4 \pi T_{c}\right)^{2} \zeta(1+\zeta) d t^{2}+\frac{d \zeta^{2}}{\zeta(1+\zeta)}\right]+d \vec{x}^{2}+\ldots
$$

where I omitted higher-order terms in $T_{c}$.

The electrostatic potential reads

$$
\Phi=\frac{2 \pi \rho T_{c}}{3} \zeta+\ldots
$$

and the field equation for the scalar field $\Psi$ near the horizon becomes

$$
\zeta(1+\zeta) \Psi^{\prime \prime}+(2 \zeta+1) \Psi^{\prime}+\frac{1}{3}\left[1+q^{2} \frac{\zeta}{1+\zeta}\right] \Psi=0
$$

where prime denotes differentiation with respect to $\zeta$.

The acceptable solution is

$$
\Psi(\zeta)=(1+\zeta)^{-i q / \sqrt{3}} F\left(-\delta_{+}-\frac{i q}{\sqrt{3}},-\delta_{-}-\frac{i q}{\sqrt{3}} ; 1 ;-\zeta\right)
$$


arbitrarily normalized so that at the horizon $\Psi(0)=1$. The other solution is discarded because it has a logarithmic singularity at the horizon.

It is easily deduced from the identity

$$
F(\alpha, \beta ; \gamma ; x)=(1-x)^{\gamma-\alpha-\beta} F(\gamma-\alpha, \gamma-\beta ; \gamma ; x)
$$

that $\Psi$ is real.

The two expressions (5.3) and (5.11), which are valid far from and near the horizon, respectively, should be matched in the overlap region. To this end, let $\zeta \rightarrow \infty$ in the near-horizon region. We obtain

$$
\Psi \approx \frac{\Gamma\left(-1-2 \delta_{+}\right)}{\Gamma\left(-\delta_{+}+\frac{i q}{\sqrt{3}}\right) \Gamma\left(-\delta_{+}-\frac{i q}{\sqrt{3}}\right)} \zeta^{\delta_{-}}+\text {c.c. }
$$

This is to be compared with the $z \rightarrow 1$ limit of the expression (5.3) which is valid away from the horizon,

$$
\Psi \sim A\left(1-z_{+}\right)^{\delta_{-}}\left(\frac{1-z_{-}}{1-z_{+}}\right)^{\frac{2 \sqrt{2}-i}{2 \sqrt{3}} q}(1-z)^{\delta_{+}}+\text {c.c. }
$$

The two expressions (5.13) and (5.14) agree in the overlap region, provided

$$
A=\frac{\Gamma\left(-1-2 \delta_{-}\right)}{\Gamma\left(-\delta_{-}+\frac{i q}{\sqrt{3}}\right) \Gamma\left(-\delta_{-}-\frac{i q}{\sqrt{3}}\right)}\left(\frac{2 \pi T_{c}}{3}\right)^{-\delta_{+}}\left(1-z_{+}\right)^{-\delta_{-}}\left(\frac{1-z_{+}}{1-z_{-}}\right)^{\frac{2 \sqrt{2}-i}{2 \sqrt{3}} q}
$$

I have thus obtained a solution to the wave equation which is parametrized by $T_{c}$. There is also an overall arbitrary multiplicative constant (since the wave equation is linear) which I am not showing. The critical temparature is fixed by imposing the remaining boundary considition at $z=0$.

For $\Delta=2$, at the boundary, we have $\Psi \sim z^{2}$, therefore

$$
A\left(-z_{+}\right)^{\delta_{-}}\left(\frac{z_{-}}{z_{+}}\right)^{\frac{2 \sqrt{2}-i}{2 \sqrt{3}} q} F\left(-\delta_{-}+2 \sqrt{\frac{2}{3}} q,-\delta_{-}-\frac{i q}{\sqrt{3}} ;-2 \delta_{-} ;-2 z_{+}\right)+\text {c.c. }=0
$$

By solving this constraint, one determines $T_{c}$. In figure $15 \mathrm{I}$ plot the scale invariant quantity $T_{c} / \sqrt{\rho}$ as a function of $q^{2}$. For $\Delta=1$, the constraint to be solved is similar to (5.16) and is obtained by demanding $\Psi \sim z+\mathscr{O}\left(z^{3}\right)$ near the boundary. Notice that $T_{c} \rightarrow 0$ as $q^{2} \rightarrow-\frac{1}{4}$. To unerstand the physical relevance of negative $q^{2}$, write the complex scalar in terms of real scalars as $\frac{1}{\sqrt{2}} \Psi e^{i q \theta}$. Then the action reads

$$
S_{\text {scalar }}=\frac{1}{2} \int d^{4} x \sqrt{-g}\left[\partial_{\mu} \Psi \partial^{\mu} \Psi+q^{2} \Psi^{2}\left(\partial_{\mu} \theta-A_{\mu}\right)^{2}-m^{2} \Psi^{2}\right]
$$

Thus, $\theta$ is a Stückelberg field giving mass to the vector potential $A_{\mu}$ when $\Psi$ condenses. One can fix the gauge by setting $\theta=0$. Then $q^{2}<0$ implies a negative mass which would normally signal an instability. However, there is no instability in AdS space as long as one stays above the BF bound. Also notice that for $q=0, \theta$ decouples. It would be interesting to explore the physical consequences in this case.

For a general $\mathrm{m}^{2}$,

$$
q^{2} \geq q_{c}^{2} \equiv \frac{3+2 m^{2}}{4}
$$



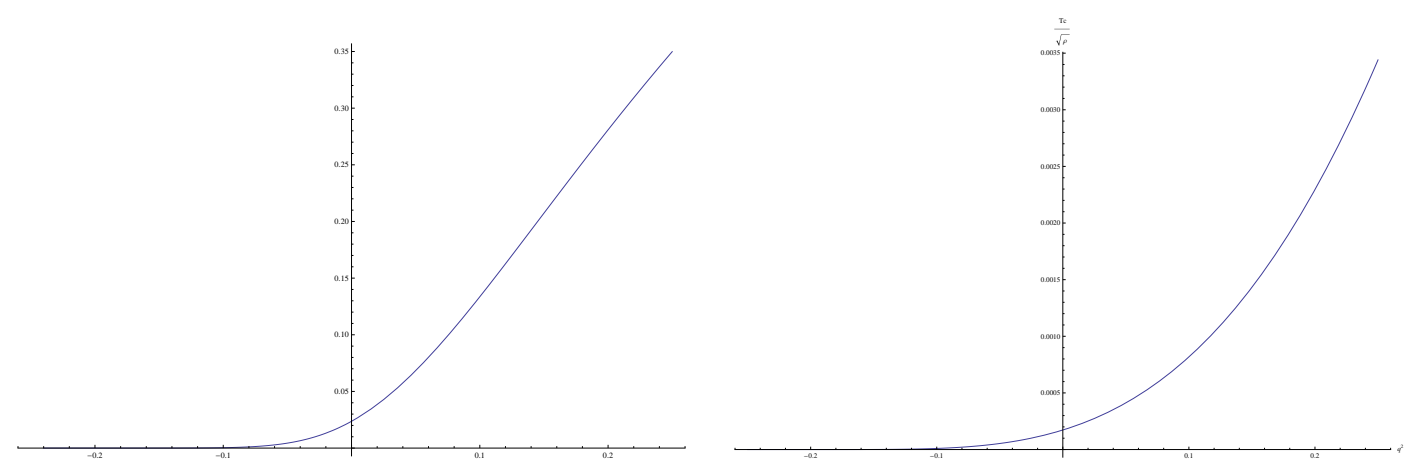

Figure 15: The critical temperature $v s . q^{2}$ for $\Delta=1$ (left panel) and $\Delta=2$ (right panel) [8].
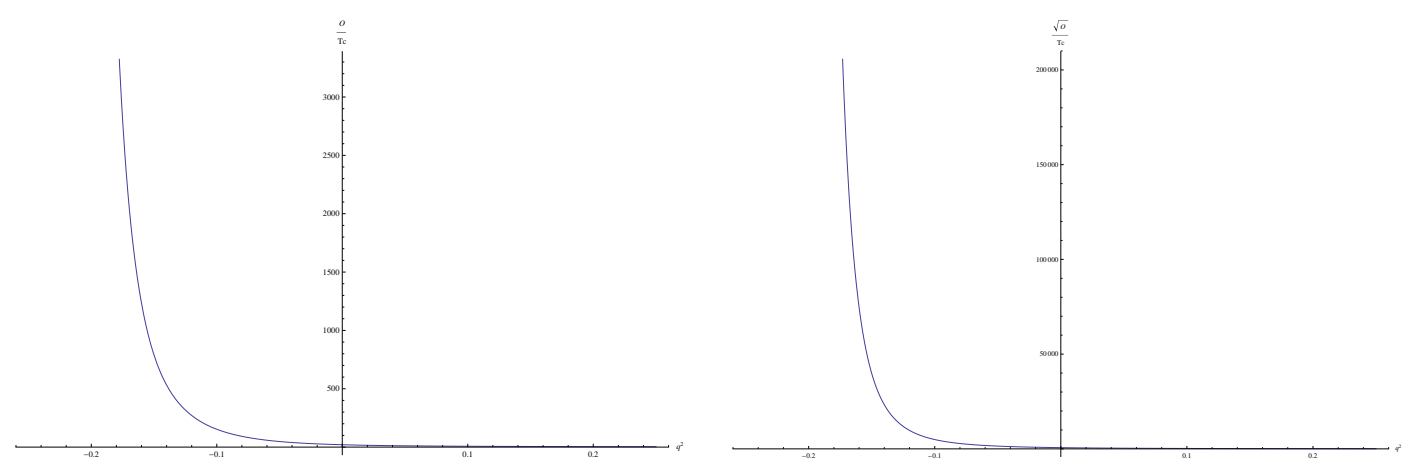

Figure 16: The energy gap vs. $q^{2}$ for $\Delta=1$ (left panel) and $\Delta=2$ (right panel) [8].

Below $T_{c}$, one can use iteration to solve the field equations, as in the probe limit. The results for the energy gap are shown in figure 16. Evidently, the energy gap in units of $T_{c}$ diverges as we approach the critical point $\left(q^{2} \rightarrow q_{c}^{2}\right)$ and $T_{c} \rightarrow 0$. This should be contrasted with the value 3.4 obtain in BCS theory and $\sim 8$ for $\Delta=2$ in the probe limit $\left(q^{2} \rightarrow \infty\right.$, see fig. 3).

\section{Conclusion}

Gravity is believed to provide a dual description of superconductors at strong coupling through holography (holographic superconductors). I used analytic techniques to probe low temperature physics where numerical instabilities arise, in order to understand the ground state of these systems. In the probe limit (large $q^{2}$, where $q$ is the charge of the scalar field that condenses), the energy gap diverges for scaling dimensions below the BF bound. I showed analytically that at the BF bound the energy gap also diverges (albeit very mildly), which is not easily seen numerically. I calculated the conductivity analytically and showed that, as a function of the frequency, it has an increasing number of spikes as one lowers the temperature at the BF bound. The positions of these spikes are determined by the zeroes of the Airy (digamma) function in $2+1(3+1)$ dimensions. I also found an analytic relation between the energy gap $E_{g}$ and the frequency gap in the conductivity $\omega_{g}$ which differs from the weak coupling (BCS) result $E_{g}=\omega_{g} / 2$ in condensed matter theory.

At the other end of the range of $q^{2}$ (near extremality), corresponding to large chemical potential, I developed analytic tools to probe the $T_{c} \rightarrow 0$ limit and found a quantum critical point at 
$q^{2}=q_{c}^{2}$ where the energy gap diverges. It would be interesting to analyze this limit further in order to elucidate the underlying physical mechanism of the instability.

A lot of work remains to be done toward a more realistic description, e.g., by incorporating spatial dependence to account for the lattice structure, pseudogap, doping, etc.

\section{References}

[1] J. M. Maldacena, The large N limit of superconformal field theories and supergravity, Adv. Theor. Math. Phys. 2 (1998) 231 [hep-th/9711200].

[2] S. S. Gubser, Breaking an Abelian gauge symmetry near a black hole horizon, Phys. Rev. D78 (2008) 065034 [arXiv:0801.2977 [hep-th]].

[3] S. A. Hartnoll, C. P. Herzog and G. T. Horowitz, Building a Holographic Superconductor, Phys. Rev. Lett. 101 (2008) 031601 [arXiv: 0803.3295 [hep-th] ].

[4] G. T. Horowitz and M. M. Roberts, Holographic Superconductors with Various Condensates, Phys. Rev. D78 (2008) 126008 [arXiv: 0810.1077 [hep-th]].

[5] S. Franco, A. Garcia-Garcia and D. Rodriguez-Gomez, A general class of holographic superconductors, JHEP 1004 (2010) 092 [arXiv:0906.1214 [hep-th] ].

[6] G. Siopsis and J. Therrien, Analytic calculation of properties of holographic superconductors, JHEP 1005 (2010) 013 [arXiv: 1003.4275 [hep-th] ].

[7] G. Siopsis, J. Therrien, and S. Musiri, Holographic superconductors near the Breitenlohner-Freedman bound, Class. Quant. Grav. 29 (2012) 085007 [arXiv:1011.2938 [hep-th] ].

[8] J. Alsup, G. Siopsis, and J. Therrien, Hair on near-extremal Reissner-Nordström AdS black holes, arXiv: 1110.3342 [hep-th].

[9] G. T. Horowitz and M. M. Roberts, Zero Temperature Limit of Holographic Superconductors, JHEP 0911 (2009) 015 [arXiv:0908.3677 [hep-th]].

[10] T. Hertog and K. Maeda, Stability and thermodynamics of AdS black holes with scalar hair, Phys. Rev. D71 (2005) 024001 [hep-th/ 0409314$].$

[11] G. Koutsoumbas, E. Papantonopoulos and G. Siopsis, Exact Gravity Dual of a Gapless Superconductor, JHEP 0907 (2009) 026 [arXiv: 0902.0733 [hep-th] ].

[12] S. A. Hartnoll, C. P. Herzog and G. T. Horowitz, Holographic Superconductors, JHEP 0812 (2008) 015 [arXiv:0810.1563 [hep-th]]. 